\title{
Axially symmetric hollow beams using refractive conical lenses
}

\author{
M. de Angelis ${ }^{\mathrm{a}, *}$, L. Cacciapuoti ${ }^{\mathrm{b}}$, G. Pierattini ${ }^{\mathrm{a}}$, G.M. Tino ${ }^{\mathrm{c}}$ \\ ${ }^{a}$ Istituto di Cibernetica del CNR, via Toiano 6, I-80072 Arco Felice, Italy \\ ${ }^{\mathrm{b}}$ Dipartimento di Fisica, Università degli Studi di Firenze, Largo E. Fermi 2, I-50125 Firenze, Italy \\ 'Dipartimento di Scienze Fisiche, Università degli Studi di Napoli "Federico II" and INFM, \\ Complesso Universitario di Monte S.Angelo, via Cintia, I-80126, Italy
}

Received 20 December 2000; received in revised form 23 April 2001; accepted 24 April 2001

\begin{abstract}
We explore the possibility of producing several beam shapes with conical or cylindrical geometry using conical lenses. We discuss optical schemes for generation of hollow laser beams; with 3D intensity distribution surrounding all directions, a dark core region can be obtained. These optical fields are important for the confinement of cold atoms in 2D or 3D blue dipole potentials.

(C) 2002 Published by Elsevier Science Ltd.
\end{abstract}

Keywords: Axicon; Optical dipole trap

The conical lens was first used by McLeod [1]; in his work he mentioned the word axicon to describe any refractive or diffractive optical element with a revolution symmetry which images a point source on its axis over a range of points along its axis. There are many different kinds of axicons, but probably the most important one is the conical lens. Axicons have been used in several applications as optical element in telescopes for long-range alignment [1], in shearing interferometer with radial displacement [2], for annular focusing in laser machining [3], for the generation of intense non-diffracting beams [4,5].

A conical lens has been recently used to produce a hollow laser beam for application in atom trapping [6] and atom guiding [7]. In the field of optical confinement of cold atoms, hollow laser beams have attracted increasing interest (for a review on optical dipole traps see [8]). Optical dipole traps rely on the interaction of

*Corresponding author. Tel.: + 39-081-853-4152; fax: + 39-081-526-7654.

E-mail address: m.deangelis@na.infn.it (M. de Angelis). 
the atomic electric dipole moment with a far-detuned radiation field. The trapping potential originates from the light shift induced on atomic levels by the light field. The potential is attractive towards high intensity field region for frequency light lower than the unperturbed frequency transition (red-detuned optical trap) and repulsive in the opposite case (blue-detuned optical trap). Long trap coherence times and very little perturbations on atomic levels can be achieved in blue-detuned optical dipole traps rather than in red-detuned optical traps. The main problem in the bluedetuned trap is the realization of a laser beam geometry, where a dark region is surrounded by light in all directions. Different methods have been developed to produce hollow laser beam geometries using refractive [9], or holographic [10] techniques for optical confinement in two dimensions; two diffractive methods have been recently realized for the three dimensional confinement $[11,12]$ : using a single laser beam they generate a dark region surrounded in each direction by regions of high light intensity; this kind of configuration is usually called "bottle beam" trap. Unfortunately, diffractive optical elements have two drawbacks: they have a limited transfer efficiency (around 50\%) of the input light and it is very difficult to change in situ the geometry of the beam which is very suitable in trapping cold atoms.

We have studied the generation of conical, cylindrical and "bottle" shaped beams using conical lenses in combination with spherical lenses. The experimental set-ups are very simple and offer the possibility to change the beam geometry in situ as shown in [13]. Moreover, our configuration gives the possibility to use only one single laser beam to generate the repulsive walls in two or three dimensions. The method uses only refractive optics which guarantee a slight dependence on the wavelength of the light field and a $100 \%$ efficiency of the input power transfer.

In Fig. 1(a), the section of a conical lens is shown. It is like a biprism with refractive index $n$ and refractive angle $\alpha$. If the conical lens is illuminated by a collimated laser beam (on an axis point source at infinity), then the incident rays at a distance $r_{1}$ from the axis intersect at some point $z$ along the axis; those at another position $r_{2}$ intersect at another value of $z$, and so on. The conical lens is generated by the rotation of the prism of Fig. 1(a); this produces an image that is focused at all positions to its right, up to a maximum distance. This maximum distance is called focal range and is determined by the axicon aperture, its base angle and its refractive index. Far from the axicon, it is possible to observe a conical shadow; when the thin axicon approximation is valid $(\alpha \ll 1)$, its divergence angle is $2 \beta=2 \alpha(n-1)$. It is evident that the conical lens is an excellent element for generating hollow beams. By illuminating a conical lens with a collimated light field it is possible to obtain a collimated beam with conical geometry which propagates with a divergence angle $2 \beta$. A spherical lens placed before the axicon can be used to focus the conical beam as shown in [6]; it is possible to see (Fig. 1(b)) that at the rear focal plane of the lens the refracted rays converge to a ring of radius

$$
R_{0}=\alpha(n-1)(f-d),
$$

where $d$ is the distance between the focusing lens and the axicon and $f$ is the lens focal length. In order to determine the optical field generated by a system lensaxicon, consider a laser beam having a wave vector $k=2 \pi / \lambda$, where $\lambda$ is the field 


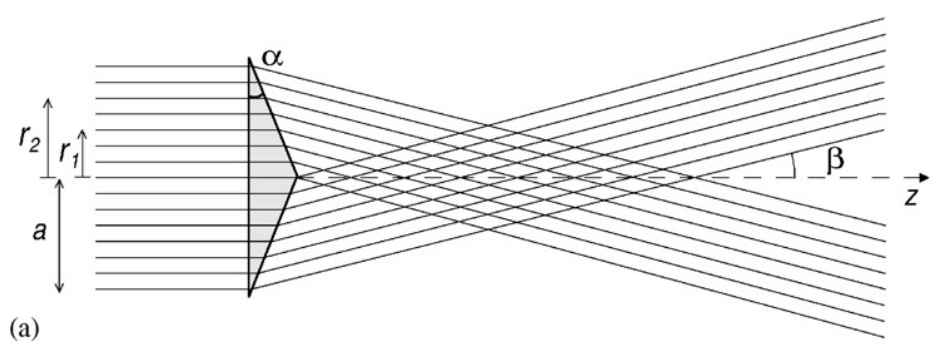

(a)

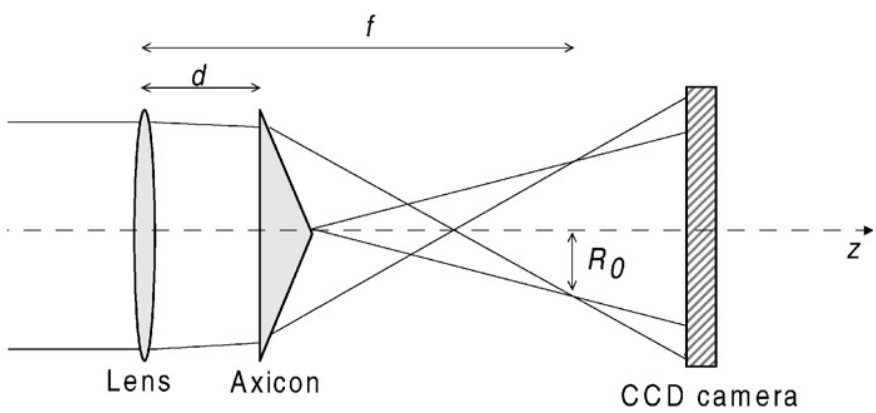

(b)

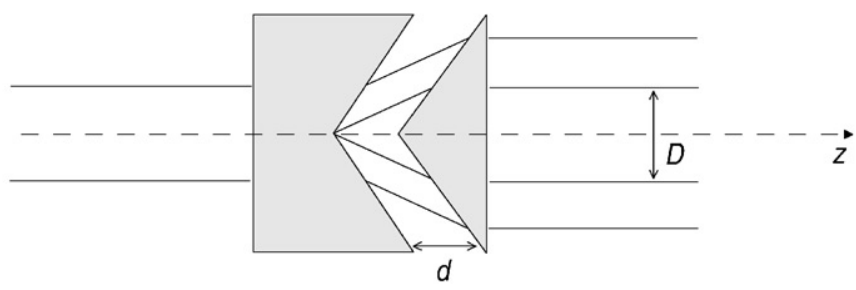

Fig. 1. (a) Linear axicon. (b) Propagation of a collimated beam through a lens-axicon doublet. The radius of the light ring is $R_{0}=\alpha(n-1)(f-d)$, where $f$ is the focal length. (c) Production of a cylindrical beam using an input divergent conical lens and an output convergent conical lens.

wavelength, and a Gaussian amplitude profile $U_{0}(\rho)$ illuminating the lens axicon combination. The Gaussian amplitude distribution at the lens aperture is given by the equation

$$
U_{0}(\rho)=\sqrt{I_{0}} \exp \left(-\frac{\mathrm{i} k \rho^{2}}{2 q_{0}}\right),
$$

where $q_{0}$ is the complex curvature radius of the Gaussian beam

$$
\frac{1}{q_{0}}=\frac{1}{R}-\frac{2 \mathrm{i}}{k w_{0}^{2}}
$$

and where $R_{0}$ is the curvature radius of the wavefront and $w_{0}$ the radius at $1 / e^{2}$ of the intensity distribution. When the Gaussian beam passes through the focusing thin lens with focal length $f$ and propagates through a distance $d$, the complex curvature 
radius $q$ changes following the imaging equation [14]:

$$
q=\frac{(1-d / f) q_{0}+d}{1-q_{0} / f} .
$$

To evaluate the output intensity profile in a point of cylindrical coordinates $(r, z)$ after the cone, the usual Kirchoff-Fresnel integral [15] for a thin axicon and an aperture radius $a$ can be used

$$
U(r, z)=\int_{0}^{a} U_{\mathrm{A}}(\rho) \exp \left(-\mathrm{i} k \frac{\rho^{2}}{2 z}\right) J_{0}\left(\frac{k r \rho}{z}\right) \rho \mathrm{d} \rho,
$$

where $\rho$ is the radial coordinate on the flat surface of the cone and $J_{0}$ is the zerothorder Bessel function. The amplitude distribution on the aperture $U_{\mathrm{A}}(\rho)$ is given by the product of the incident Gaussian beam distribution at the flat surface of the conical lens with the phase factor $\exp [+\mathrm{i} k(n-1) \alpha \rho]$ introduced by the propagation in the axicon itself, namely

$$
U_{\mathrm{A}}(\rho)=\sqrt{I_{0}} \exp \left\{-\mathrm{i} k\left[\frac{\rho^{2}}{2 q}-(n-1) \alpha \rho\right]\right\} .
$$

The diffraction integral expressed in Eq. (5) can be easily numerically evaluated and, in case a lens-axicon combination is used, the intensity distribution of the light of the generated two dimensional trap can be estimated.

In the experimental set-up used to study the conical beam properties, the laser beam is produced by a diode laser $(\lambda=0.780 \mu \mathrm{m})$. The output beam is symmetrized by an anamorphic prism pair, spatially filtered with a $50 \mu \mathrm{m}$ diameter pin hole and expanded in order to obtain a beam with $\mathrm{a} \simeq 1.5 \mathrm{~mm}$ waist. We used a refractive right circular cone realized in BK7 (refractive index $n=1.51$ at $0.780 \mu \mathrm{m}$ ) with base angle $\alpha \simeq 100 \mathrm{mrad}$; it is used in combination with a plano-convex spherical lens with focal length $f=100 \mathrm{~mm}$. The intensity distribution profile of the hollow beam is recorded using a CCD camera (array of $512 \times 512$ pixels, pixel size $11 \times 11 \mu \mathrm{m}$ ). In Fig. 2, the ring light distribution is shown in the focal plane of the lens for the distance between lens and axicon $d=85 \mathrm{~mm}$; the CCD camera is placed in the back focal plane of the spherical lens. The ring radius increases with the distance from the focal plane (see Eq. (1)). The radial sections along the two orthogonal axis show the good quality of the hollow beam. In order to estimate the ratio between light intensity in the dark region and in the light region a measurement of the light intensity scattered into the dark region should be suitable; unfortunately, it is limited by the noise of the CCD when the field intensity is increased. The transverse intensity distribution of the light ring has been estimated using a numerical evaluation of the diffraction integral expressed in Eq. (5) and the theoretical radial profile is compared to the experimental distribution in Fig. 3.

A collimated hollow beam for atom guiding has been realized in [7]. In that paper they observed that a hollow beam generated by an axicon and a spherical lens has different focal points for the inner and the outer walls; to overcome this problem, additional axicons are required which complicate the experiment. A cylindrical shaped beam with a very good collimation can be easily realized by using a doublet 

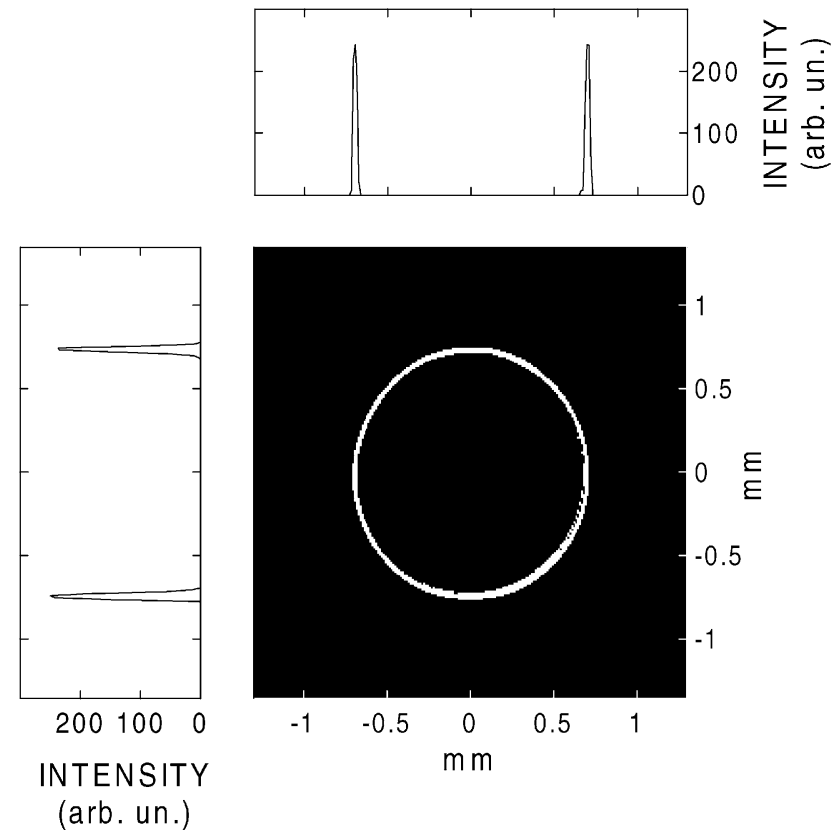

Fig. 2. Image of the ring intensity distribution in the back focal plane of the lens with distance $d=85 \mathrm{~mm}$ between lens and axicon, together with cross section of the beam profile along the vertical and horizontal axis.

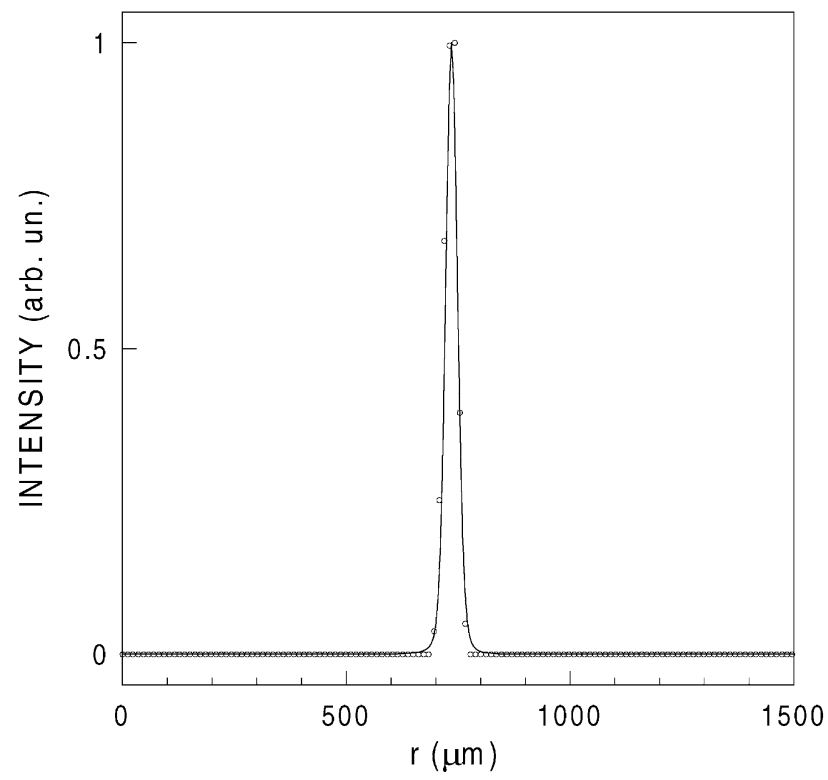

Fig. 3. Detail of the radial intensity distribution of the ring shaped image of Fig. 2 (circles); it is compared to the numerical profile given by the Fresnel-Kirchoff diffraction integral (continuous line). 
of two conical lenses, one concave and the other convex (see Fig. 1c). The emerging beam has a hollow cylindrical profile along the propagation direction of the laser beam. In the thin axicon approximation $(\alpha \ll 1)$, the diameter $D$ of the inner dark region is given by $D=2 d \alpha(n-1)$, where $d$ is the separation between the two conical lenses. The ring diameter can be changed continuously by changing the distance between the two axicons. We have used two conical lenses (a divergent one and a convergent one) with the same base angle $\alpha=200 \mathrm{mrad}$. When they are perfectly in contact, the beam profile is unaffected; if we slightly separate the two lenses, then a dark region is formed in the centre of the beam profile and a cylindrical beam is generated. Cross section of the beam is shown in Fig. 4 for a distance between the two cones $d \cong 5 \mathrm{~mm}$. In the figure, circular fringes are visible; they are generated by the Fresnel diffraction from the conical lens, as explained in [16]. The divergent and convergent axicons are plexiglass (refractive index $n=1.49$ ) home made cones and the non-perfect machining of the cone apexes generates diffraction and modulation of the profile of the mentioned circular fringes.

When a conical or cylindrical shaped beam is used for atom trapping, a restoring force along the symmetry axis is needed. In our set-ups the restoring force along the $z$-axis can be obtained by adding two laser beams or by crossing two of such hollow laser beams. For example, in Fig. 5a, the case of a 3D trap obtained using a cylindrical beam is shown.

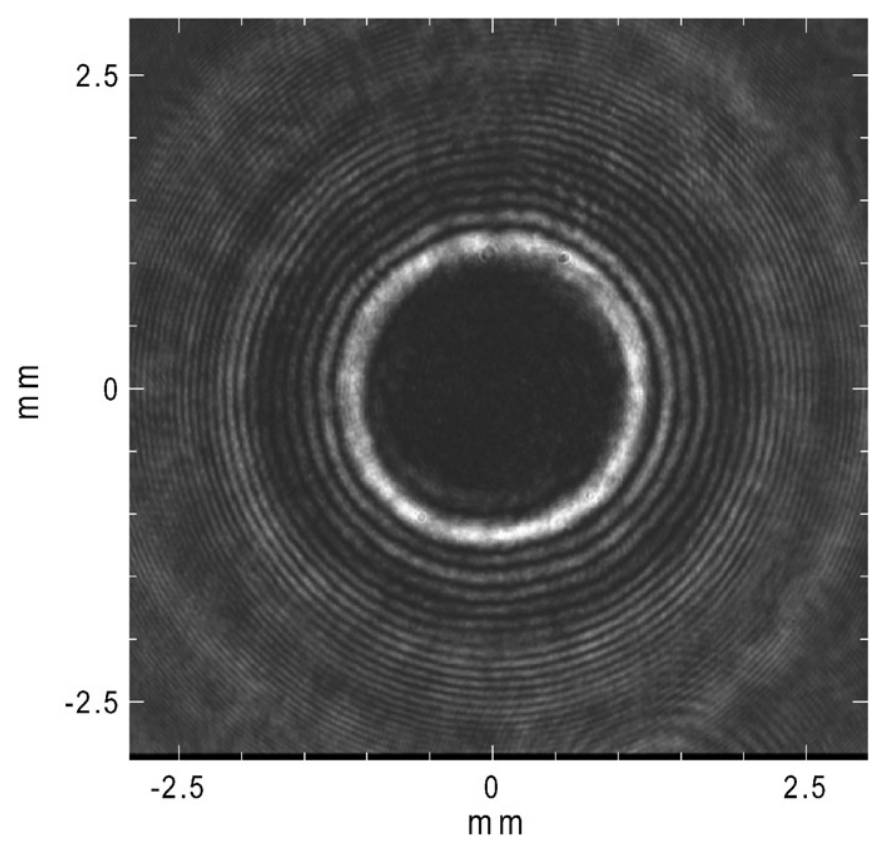

Fig. 4. Section of the cylindrical beam generated by a combination of a divergent and a convergent $200 \mathrm{mrad}$ base angle conical lenses at distance $5 \mathrm{~mm}$; the circular fringes come from the Fresnel diffraction by the conical lens. 
(a)
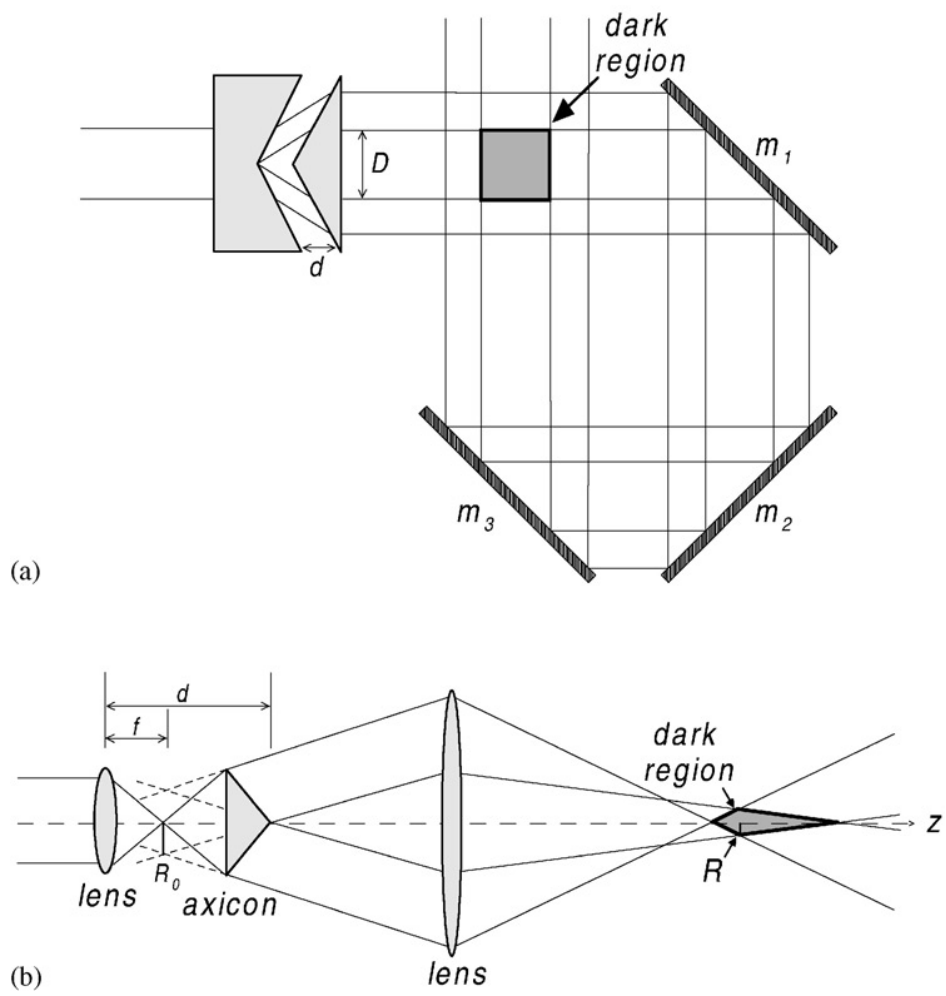

Fig. 5. (a) Schematic of the experimental arrangement for a 3D trap using a cylindrical hollow beam. (b) Schematic of the experimental arrangement for the generation of a "bottle beam". The virtual ring produced by the first lens and the axicon is drawn. The second lens images this ring and generates the dark region.

We have also studied a scheme in order to create an optical potential bounded in all directions using a single laser beam. In the experimental set-up shown in Fig. 5b, a collimated laser beam crosses a converging lens, enters the axicon and comes out from a second converging lens. The first lens and the axicon produce a ring-shaped virtual image which is focused by the second lens. In the plane of image of the second lens it is possible to observe a dark region surrounded by light. If $G$ is the magnification produced by the second lens, then the ring shaped intensity distribution in the plane of image has a radius

$$
R=G \alpha(n-1)(d-f) .
$$

In order to investigate the propagation of the hollow beam we moved the CCD camera along the optical $z$-axis and recorded a series of beam profiles as a function of the displacement along $z$. In Fig. 6 the image of the intensity profile in grey levels is reported: in (a) the image in the transverse plane at $z=0.75 \mathrm{~mm}$ (radial distribution) is shown which has an annular shape; in (b) it is possible to see the 


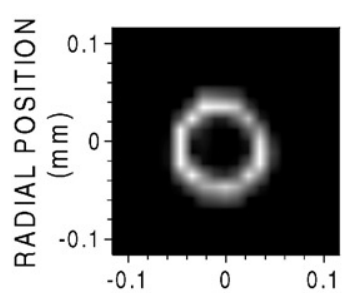

(a)

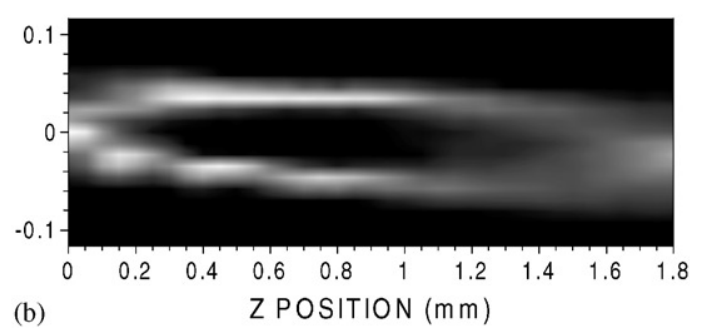

Fig. 6. Image of the intensity profile in grey levels of the optical bottle beam. (a) Intensity profile of the bottle beam in the plane of image of the second lens. (b) Image of the intensity profile of the bottle beam in a plane containing the $z$-axis.

intensity profile along the propagation direction of the laser beam (z-axis). These images show a "bottle" shaped dark region surrounded by light in all directions; this 3D blue-detuned intensity distribution provides the required repulsive walls to trap atoms in the dark region. We have realized a dark region surrounded by light with a ring diameter of $48 \mu \mathrm{m}$ and an axial length $0.960 \mathrm{~mm}$. In this configuration, the first lens is a plano-convex 1 in diameter lens with focal length $125 \mathrm{~mm}$ and the second one is a plano-convex 2 in diameter with focal length $100 \mathrm{~mm}$. The distance between the first lens and the axicon is $\sim 145 \mathrm{~mm}$ and the distance between the axicon and the second lens is $\sim 930 \mathrm{~mm}$; these distances are considered from the axicon apex to the plane surface of the lenses. The optical trap is around the focal plane of the second lens. The hollow beam provides a good $3 \mathrm{D}$ confinement only if the core region is extremely dark. We cannot measure the intensity of the scattered light in the centre of the trap because we are limited by the noise of the CCD camera in the dark region of the trap when light intensity is increased. However, it is reasonable to estimate the scattered light less than $1 \%$ compared to the peak intensity, observed in the plane of the image (see [6]).

In conclusion, we have studied the generation of conical shaped beams using a conical lens in combination with a spherical lens, and a cylindrical beam generated by a combination of a divergent and a convergent conical lens. Finally, we show a simple experimental set-up using one axicon and two spherical lenses to obtain a single laser beam with a well localized intensity null surrounded in all directions by regions of high intensity (a similar scheme was presented by Orozco [17]). In all these configurations, it is easy to change the geometry of the laser beam and the linear dimension of the dark region. These beams can be used for guiding or trapping cold atomic samples in a low intensity region, where the interaction with the light field is minimum.

\section{Acknowledgements}

The authors thank Mauro Pucci for machining the conical lens. 


\section{References}

[1] McLeod JH. The axicon: a new type of optical element. J Opt Soc Am 1954;44:592-7.

[2] Bryngdahl O. Shearing interferometry with constant radial displacement. J Opt Soc Am 1971;61: 169-72.

[3] Bélanger PA, Rioux M. Ring pattern of a lens-axicon doublet illuminated by a Gaussian beam. Appl Opt 1978;17:1080-6.

[4] Herman RM, Wiggins TA. Production and uses of diffractionless beams. J Opt Soc Amer A $1991 ; 8: 932-42$.

[5] Altucci C, Bruzzese R, D'Antuoni D, De Lisio C, Solimeno S. Harmonic generation in gases by using Bessel-Gauss laser beams. J Opt Soc Am B 2000;17:34.

[6] Manek I, Ovchinnikov YB, Grimm R. Generation of a hollow laser beam for atom trapping using an axicon. Opt Commun 1998;147:67-70.

[7] Song Y, Milan D, Hill III WT. Long, narrow all-light atom guide. Opt Lett 1999;24:1805-7.

[8] Grimm R, Weidemüller M, Ovchinnikov YB. Optical dipole traps for neutral atoms. Adv At Mol Opt Phys 2000;42:95-170.

[9] Kuga T, Torii Y, Shiokawa N, Hirano T. Novel optical trap of atoms with a Doughnut beam. Phys Rev Lett 1997;78:4713-6.

[10] Kuppens S, Rauner M, Schiffer M, Sengstock K, Ertmer W, Dorsselaer FE, Nienhuis G. Polarization-gradient cooling in a strong doughnut-mode dipole potential. Phys Rev A 1998;58:306879.

[11] Ozeri R, Khaykovich 1, Davidson N. Long spin relaxation times in a single-beam blue-detuned optical trap. Phys Rev A 1999;59:R1750-3.

[12] Arlt J, Padgett MJ. Generation of a beam with a dark focus surrounded by regions of higher intensity: the optical bottle beam. Opt Lett 2000;25:191-3.

[13] Cacciapuoti L, de Angelis M, Pierattini G, Tino GM. Single-beam optical bottle for cold atoms using a conical lens. Eur Phys J 2001;14:373-6.

[14] Kogelnik H, Li T. Laser Beams and Resonators. Appl Opt 1966;5:1550-67.

[15] Born M, Wolf E. Principles of optics. New York: Pergamon, 1980.

[16] Perez MV, Gomez-Reino C, Cuadrado JM. Diffraction pattern and zone plates produced by thin linear axicons. Opt Acta 1986;33:1161-76.

[17] Orozco LA. Poster presented at the XIV International Conference on Laser Spectroscopy, Innsbruk 1999. 